\title{
Density measurement in a laser-plasma-accelerator capillary using Raman scattering
}

\author{
T. Weineisen, ${ }^{1,2}$ B. Göppner, ${ }^{1,2}$ K. Schmid, ${ }^{1}$ M. Fuchs, ${ }^{1,2}$ H. Schröder, ${ }^{1}$ S. Karsch, ${ }^{1,2}$ and F. Grüner ${ }^{1,2, *}$ \\ ${ }^{1}$ Max Planck Institut für Quantenoptik, 85748 Garching, Germany \\ ${ }^{2}$ Department of Physics, Ludwig Maximilians Universität, 85748 Garching, Germany
}

(Received 11 September 2010; published 24 May 2011)

\begin{abstract}
Laser wakefield accelerators have shown $1 \mathrm{GeV}$ electron beams with some $10 \mathrm{pC}$ charge from centimeter-length gas capillaries. The electrons are accelerated by the field of a plasma wave trailing an intense laser pulse. For improving the stability, electron injection and acceleration should be separated. One possible scheme is self-injection with a plasma density gradient and subsequent acceleration at constant density. This can be realized by embedding a high-density gas jet into a capillary. A critical parameter for this scheme to work is the realization of a specific density gradient, therefore a robust measurement is desirable. A new method utilizing the density dependence of Raman scattering has been used to characterize the high-density region of a neutral gas within a capillary with a few ten micrometer longitudinal resolution. This allowed us to measure a density drop of a factor of 4 within 200 micrometers.
\end{abstract}

DOI: 10.1103/PhysRevSTAB.14.050705

PACS numbers: 41.60.Cr, 42.65.Dr

\section{INTRODUCTION}

Laser wakefield accelerators (LWFA)—in which electrons are accelerated by the field of a plasma wave trailing an intense laser pulse- have been demonstrated as compact sources of electrons with energies in the $\mathrm{GeV}$ range [1,2]. Discharge capillaries [3] with a few hundred micrometers in diameter can be used to shape the plasma density as well as to guide the laser over a distance longer than a Rayleigh length, thus allowing to more efficiently use the laser energy for acceleration. However, in most experiments the electron beams show instabilities in energy spread and beam pointing.

It has been proposed [4-6] that a plasma density gradient can be introduced to control the self-injection process of the electrons into the accelerating wakefield. A sudden density decrease forces the plasma wave to elongate and leads to a decrease in phase velocity. This process enhances electron trapping when the reduced phase velocity matches the plasma fluid velocity. First density down ramp experiments [7] using a gas jet have already shown high charges in the order of nC albeit with an energy spread $\Delta E / E$ around 25\% FWHM for only $1 \mathrm{MeV}$ electrons. However, this might be developed into an attractive source of electrons if the injected charge could be postaccelerated to high energy in a subsequent plasma wave without further increasing the absolute energy spread $\Delta E$, though it is not clear whether the second condition can be fully met. A first experimental approach may consist of a combination of a high- and a

\footnotetext{
*Corresponding author.

florian.gruener@physik.uni-muenchen.de

Published by the American Physical Society under the terms of the Creative Commons Attribution 3.0 License. Further distribution of this work must maintain attribution to the author(s) and the published article's title, journal citation, and DOI.
}

low-density region separated by a steep density ramp. A density shape with these two stages can be realized through embedding a free flow gas jet into a capillary, see Fig. 2(a) [6]. The gas jet provides the steep density profile and the capillary ensures a constant density afterwards.

Using femtosecond laser machining we have manufactured such a capillary with built-in free flow gas nozzle. Typical capillaries are few hundred micrometers in diameter and few centimeters in length. The nozzle and further gas inlets have a diameter of $700 \mu \mathrm{m}$. Since the laser machining process creates a rough inner surface of the capillary, diagnostics relying on phase detection are not applicable for determining the density properties in the channel.

A feedback on the longitudinal density profile is a key for controlling the interaction and for modeling it with particle in cell (PIC) simulations. Measuring the density is not straightforward because no sensors can be implemented into the capillary. Interferometry measurements as used in previous works $[8,9]$ require rectangular capillaries made from polished sapphire surfaces in order to preserve the phase of the transmitted laser beam. This method uses the large refractive index of the plasma column, which is large compared to the one of neutral gas, in order to measure the free-electron density in the plasma. Furthermore, it allows measurements of the radial density distribution as well as time resolved measurements of the plasma. Laser-machined capillaries do not have optical transparent walls, thus interferometry is not a suitable method to quantify a built-in gas jet.

Here we present for the first time a method to characterize the density gradient of a neutral gas in a laser-machined capillary. The density dependence of Raman scattering is used to obtain the signal from inside the capillary. Therefore a high power $\mathrm{cw}$ laser is focused through the nitrogen-filled capillary. The excited diatomic gas emits Raman light which experiences a frequency shift in comparison to the incident 
light. This allows to spectrally distinguish the Raman photons from pump photons scattered on the walls for instance. The Raman signal is recorded perpendicular to the laser and the capillary. This method allows explicitly measuring a geometry that does not require plan parallel walls like the interferometry and is, hence, well suited for the proposed geometry of an embedded gas jet.

\section{EXPERIMENT}

\section{A. Capillary production and setup}

The capillaries are manufactured using a laser ablation technique. An $80 \mathrm{fs}$ laser pulse is focused to a spot size of $7 \mu \mathrm{m}$ at $1 / e^{2}$ (intensity) on the sapphire surface with a repetition rate of $1 \mathrm{kHz}$ at $20 \mu \mathrm{J}$ per shot. A series of shots, each producing a crater of $3 \mu \mathrm{m}$ depth and a diameter of $7 \mu \mathrm{m}$, can machine any desired 3D geometry. Figure 2(a) shows the capillary used in the experiment. Only the capillary with nozzle was laser machined to provide reproducible flow properties, while inlets and outlets are diamond ground due to the faster fabrication time. The gas supply pipes as well as the vacuum outlets were diamond ground because of the time-consuming laser machining process. The capillary has a width of $300 \mu \mathrm{m}$ on the surface and reduces to $145 \mu \mathrm{m}$ on the bottom, the nozzle has a width of $700 \mu \mathrm{m}$, and the vacuum outlet above the nozzle expands from 1400 to $2100 \mu \mathrm{m}$ with an angle of $45^{\circ}$, all with constant depth of $230 \mu \mathrm{m}$.

A blank plate is mounted on the machined plate which provides capillary as well as inlets and outlets. This capillary was operated in vacuum (chamber pressure 0.1 to 1 mbar), see Fig. 1(a). The capillary has two gas junctions, one which is labeled "Nozzle Inflow" to the left of Fig. 2(a) and one labeled "Inlet" located to the right. The pressure at the nozzle and the gas inlet could individually be set up to 1600 mbar in steps of 1 mbar \pm 0.5 mbar. An additional vacuum pump was connected to the outlet above the nozzle. Simulations show that $85 \%$ of the gas leaves the top outlet, thus it was advisable not to lead the gas stream into the evacuated chamber but into a pump.

The nitrogen molecules inside the capillary were excited by a 6 Watt $532 \mathrm{~nm}$ VERDI V6 laser. The laser had a beam diameter of $2.25 \mathrm{~mm} \pm 10 \%$ at $1 / e^{2}$ (intensity) and was focused with an $f=25 \mathrm{~mm}$ lens through the capillary. The spot size was $118 \mu \mathrm{m}$ and the Rayleigh length was $l_{R} \approx 20 \mathrm{~mm}$, which is 3 times the length of the capillary.

\section{B. Raman scattering and measurement procedure}

The Raman scattering signal $P_{S}$ depends linear [10] on the number of molecules $N$ and, hence, the density is given by

$$
P_{S}=N \cdot \sigma_{S} \cdot I_{L}
$$

with the molecule's scattering cross section $\sigma_{S}$ and the laser intensity $I_{L}$. Raman scattering, in contrast to Rayleigh scattering, is inelastic scattering. Energy is stored in a vibrational or rotational mode of a molecule, resulting in a wavelength shift of the scattered photon. This wavelength shift is distinct for different molecules, where nitrogen has a wavelength shift of $2329 \mathrm{~cm}^{-1}$. Excited with $532 \mathrm{~nm}$, the Raman signal is at $607.2 \mathrm{~nm}$. It is recorded perpendicular to the incident light. The Raman differential scattering cross section for nitrogen at $532 \mathrm{~nm}$ reads $\sigma_{S} / d \Omega=$ $4.0 \times 10^{-31} \mathrm{~cm}^{2} / \mathrm{sr}$ [11]. This is a factor of 1600 below the Rayleigh signal. Raman scattering is not 4- $\pi$ symmetrical for linear polarized light—-this, however, has not been

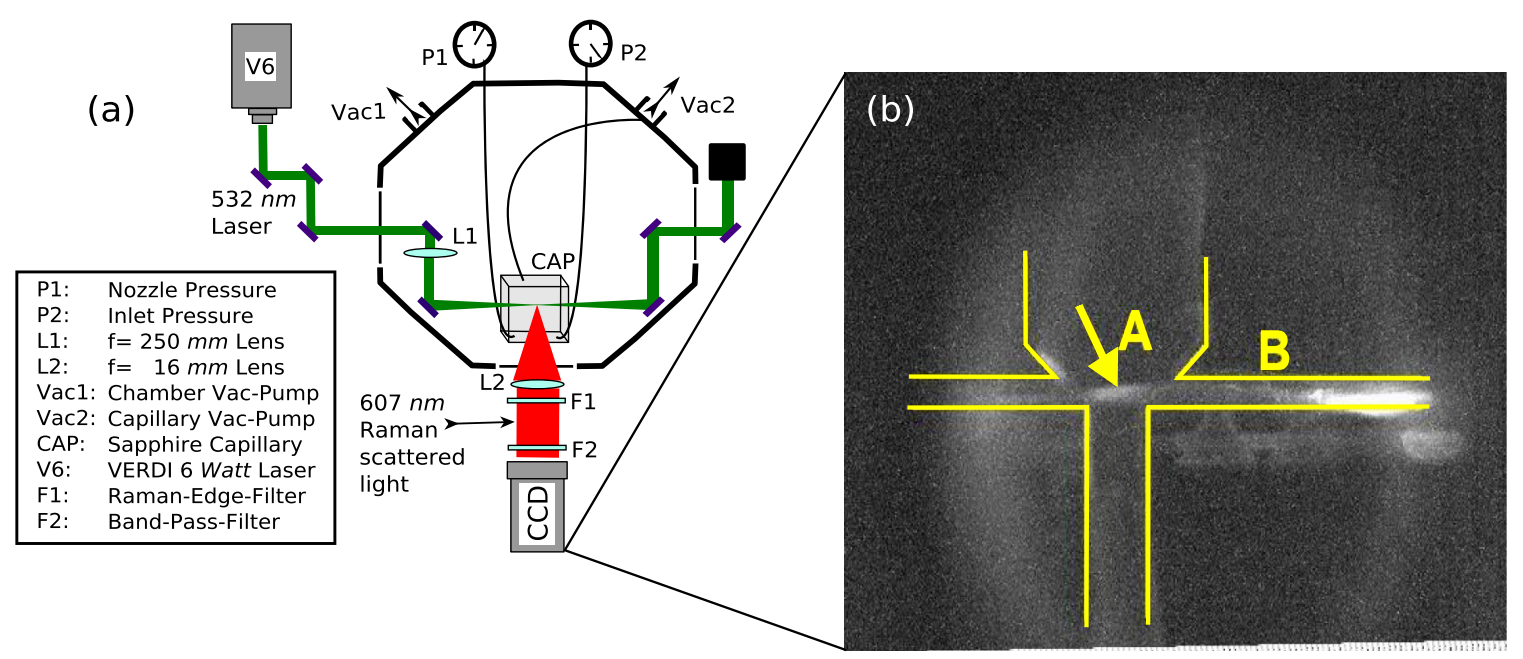

FIG. 1. (a) Top view of the experimental setup. A $532 \mathrm{~nm}$ laser (green) is focused by an $f=250 \mathrm{~mm}$ lens (L1) into the sapphire capillary (CAP). The gas density and profile in the capillary is set by two separate pressure valves that lead to each gas inlet, see Fig. 2(a). Raman scattered light (red) is filtered and imaged into a CCD. (b) The image shows the Raman signal, the lines are additionally drawn and indicate the capillary. The increased signal in nozzle region A is due to the high-density gas jet. The stray light to the right of $\mathrm{B}$ inside the capillary is caused by the laser hitting the wall. This light was too intense for the filters. The shutter time was $7 \mathrm{~s}$. 


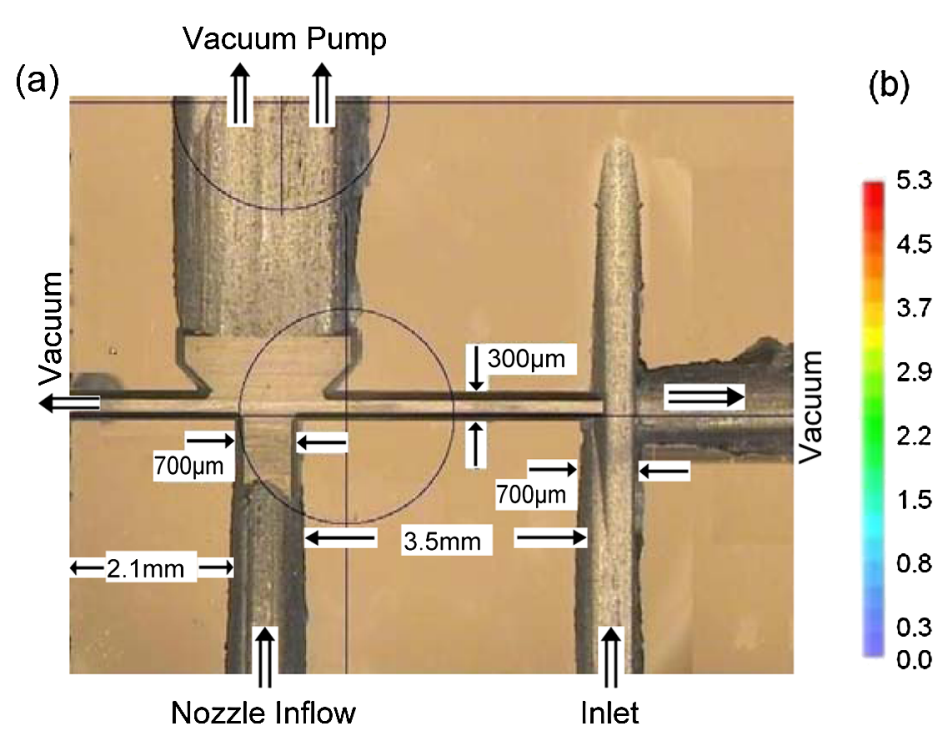

(b)

FIG. 2. Part (a) shows a photograph of the laser-machined capillary with nozzle and the diamond ground gas inlets and outlets. Part (b) illustrates the geometry of the capillary used in FLUENT simulations. The colors denote the number density in $10^{19} \times \mathrm{cm}^{-3}$. The narrow channel in the middle denotes the capillary; the boxes on the left and right side as well as on top are used to simulate vacuum. The two pipes coming from the bottom are the gas inlets for the nozzle (left) and the inlet (right).

taken into account since the detecting angle was not changed throughout the whole process.

The density dependence and the frequency shift make Raman scattering a feasible method to measure the gas density inside a sapphire capillary.

The Raman photons were collected by an imaging system, consisting of a lens, filters, and a camera objective. An $f=16 \mathrm{~mm}$ high numerical aperture $(N A=0.73)$ lens imaged the capillary/gas-jet intersection region onto a CCD camera. Its $9.9 \mu \mathrm{m}$ pixels gave a $12.1 \mu \mathrm{m} \pm$ $0.6 \mu \mathrm{m}$ resolution in the object plane. A Raman edge filter (OD 6) was used to block the green stray light and all shorter wavelengths. In addition to this stray light there was a high intensity background Raman signal generated in the sapphire bulk with its peak at $693 \mathrm{~nm}$. This signal was 3 to 4 orders of magnitude larger than the desired
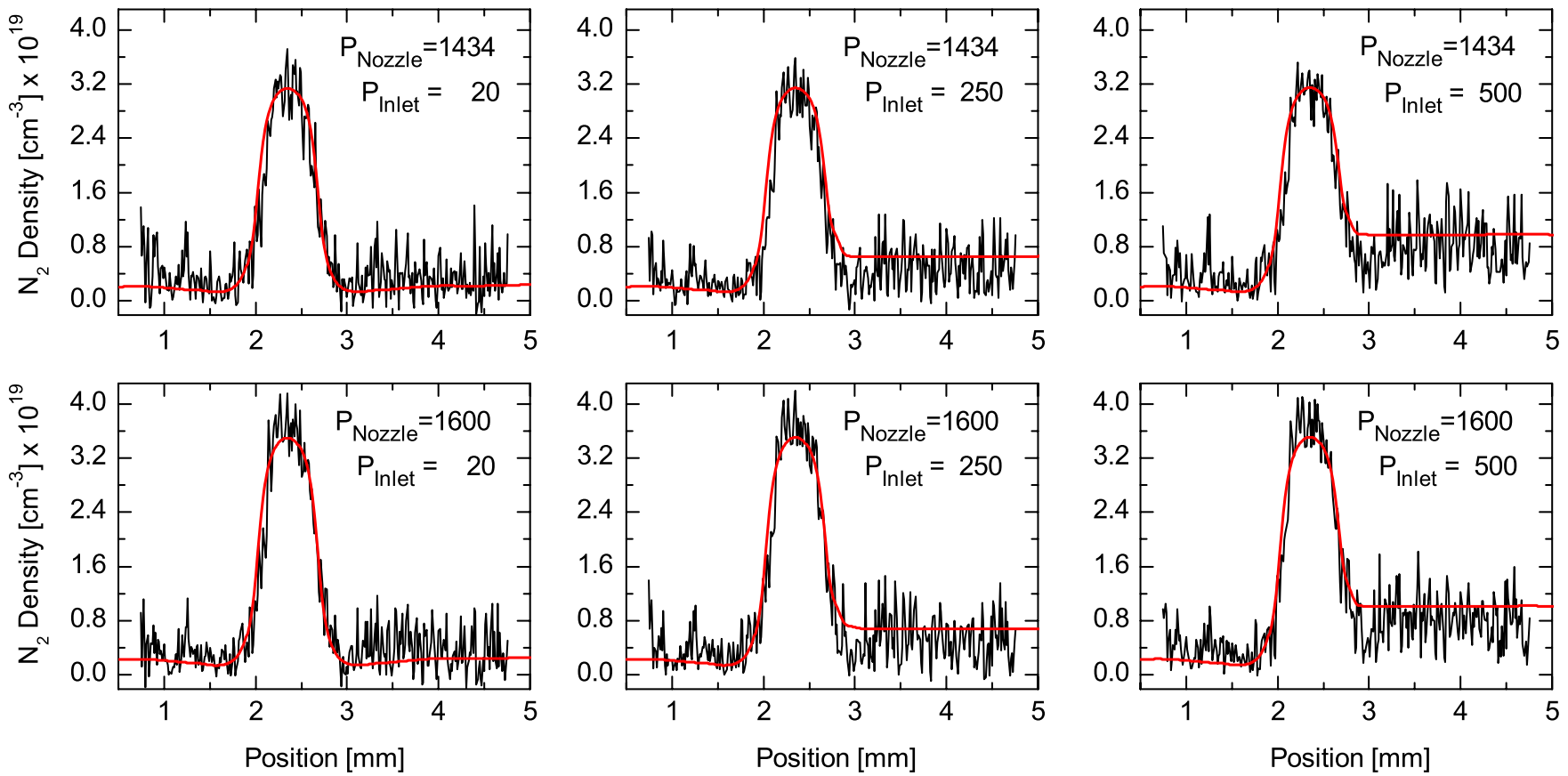

FIG. 3. Six density graphs with varying inlet and nozzle pressures (in mbar). The black graphs denote the measurements and the red graphs denote the simulation results. The $y$ axis shows the nitrogen density. The $x$ axis refers to the position along the capillary. The capillary entrance is located at $0 \mathrm{~mm}$. 
TABLE I. List of all pressure combinations (in mbar) which were measured.

\begin{tabular}{lrrrr}
\hline \hline $\mathrm{P}_{\text {nozzle }}$ & 1000 & 1200 & 1434 & 1600 \\
\hline \multirow{3}{*}{$\mathrm{P}_{\text {inlet }}$} & 13 & 17 & 20 & 20 \\
& 250 & 250 & 250 & 250 \\
& 500 & 500 & 500 & 500 \\
\hline \hline
\end{tabular}

$\mathrm{N}_{2}$-Raman signal. A bandpass filter (OD 4) was used to isolate these photons, transmitting $58 \%$ at $607.2 \mathrm{~nm}$ with a transmission width of $1.9 \mathrm{~nm}$.

In order to prove that the observed signal was in fact Raman photons and that the Raman edge filter effectively blocked the green Rayleigh and stray light, a test experiment was performed. The setup was filled in a first run with argon and in a second run with nitrogen. As argon is a noble gas which has no Raman mode, it did not show a Raman signal in contrast to the capillary filled with nitrogen. The attenuation of the laser due to, e.g., Rayleigh scattering or scattering at the walls was negligible. The axial laser intensity distribution due to focusing has been taken into account.

The shutter time of the camera was set to $7 \mathrm{~s}$. Figure 1(b) shows a nonmodified image of the Raman signal with increased scattering from the higher density in nozzle region A. The Raman photon counts are obtained from the images. For each longitudinal density lineout (see Fig. 3) four pixel rows were averaged; these four rows correspond to $50 \mu \mathrm{m}$ of the laser width. The corresponding density can be deduced by comparing the photon counts to a reference measurement. The reference was obtained by filling the whole vacuum chamber including the Raman setup to a distinct pressure in measured steps of 50 mbar up to 850 mbar; all other inlets and outlets were closed during that time. This guaranteed a constant density along the capillary. Then the same laser intensity as in the experiment is focused through the capillary and the Raman calibration signal is obtained. The reference data revealed that the signal for constant density increased slightly toward the right side, which may be caused by the strong

(a)

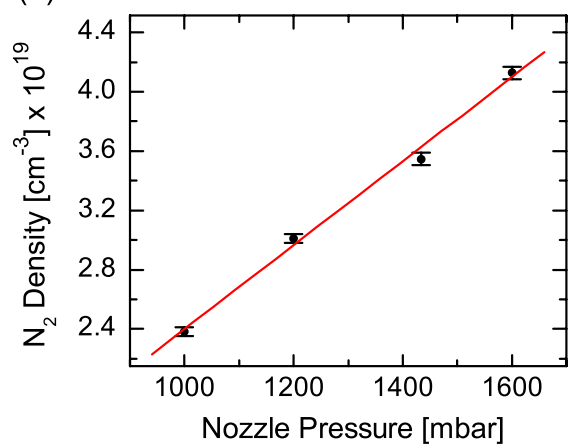

scattering at the walls in this region, see Fig. 1(b). The experimental data were matched with this rise, leading to a doubling of the noise ratio for the capillary region of $0.076 \times 10^{19} \mathrm{~cm}^{-3}$ instead of $0.031-0.043 \times 10^{19} \mathrm{~cm}^{-3}$ for the nozzle region.

\section{Simulations}

The shape of the capillary and the required pressures were determined with computational fluid dynamic (CFD) simulations. A comparison with the experimental results can be seen in Fig. 3.

The 2D calculations were carried out with the OPENFOAM 1.6 code using a transient solver suitable for trans-sonic/supersonic, laminar, or turbulent flow of a compressible gas [12]. The k-omega-SST model with wall functions was used as turbulence model. The simulated geometry seen in Fig. 2(b) has identical dimensions as the real capillary Fig. 2(a). The extension over the capillary of the right gas inlet from Fig. 2(a) creates a dead volume. This volume has no significant influence on flow properties which has been checked in simulations and was thus not discussed in further simulations.

\section{RESULTS AND DISCUSSIONS}

The pressures at the nozzle as well as at the inlet are varied; Table I gives a complete list of all 12 pressure combinations.

The densities along the capillary are plotted in the black graphs from Fig. 3. The density peaks, due to the gas jet, are well pronounced with a resolution of $12 \mu \mathrm{m}$ per pixel. The presented graphs show $4 \mathrm{~mm}$ of the $6.2 \mathrm{~mm}$ capillary with the peak density at $2.35 \mathrm{~mm}$. The peak has a FWHM of $530 \mu \mathrm{m}$ for a nozzle pressure of $1600 \mathrm{mbar}$ with inlet pressure of 250 mbar. The drop from $90 \%$ of the peak value to $10 \%$ relative to the plateau value reads $3.75 \times 10^{19} \mathrm{~cm}^{-3}$ to $0.98 \times 10^{19} \mathrm{~cm}^{-3}$ within $250 \mu \mathrm{m}$. For 1600 mbar nozzle pressure and 20 mbar inlet pressure, this drop reads $3.8 \times 10^{19} \mathrm{~cm}^{-3}$ to $0.75 \times 10^{19} \mathrm{~cm}^{-3}$ within $280 \mu \mathrm{m}$.

(b)

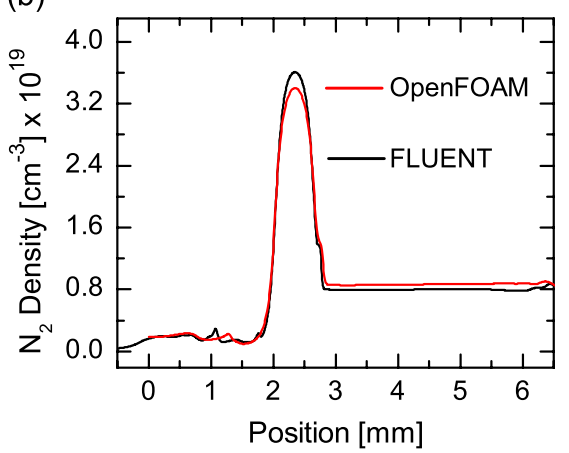

FIG. 4. (a) Applied nozzle pressure versus peak density (experimental results). The red line shows the linear fit. The error bars are RMS. (b) Comparison of an OPENFOAM simulation with a FLUENT simulation under the same conditions. 
TABLE II. List of the mean densities $\left(\times 10^{19}\left[1 / \mathrm{cm}^{3}\right]\right)$ in the capillary region $\mathrm{B}$ for all pressure combinations.

\begin{tabular}{lllll}
\hline \hline $\mathrm{P}_{\text {inlet }} \backslash \mathrm{P}_{\text {nozzle }}$ & 1000 & 1200 & 1434 & 1600 \\
\hline $13-20$ & 0.18 & 0.26 & 0.34 & 0.37 \\
250 & 0.35 & 0.45 & 0.51 & 0.54 \\
500 & 0.56 & 0.66 & 0.65 & 0.80 \\
\hline \hline
\end{tabular}

The resolution limit for the density can be computed to a root mean square (RMS) error of $0.031 \times 10^{19} \mathrm{~cm}^{-3}$ for 1000 and 1200 mbar and $0.043 \times 10^{19} \mathrm{~cm}^{-3}$ for 1434 and 1600 mbar, respectively. Each error was calculated from eight images, the density values were taken from the middle of the nozzle at $2.35 \mathrm{~mm}$. Figure 4(a) shows the peak-density versus the applied nozzle pressure with error bars. The linear fit (red line) confirms the expected linear density-pressure dependence from Eq. (1).

The experimental data reveal a density dip in the intersection of nozzle region A and capillary region B at approximately 2.8 to $3.3 \mathrm{~mm}$. This dip cannot be seen in simulations.

The mean density values from capillary region B are listed in Table II, with an RMS error of $0.076 \times 10^{19} \mathrm{~cm}^{-3}$. The red graphs in Fig. 3 denote the density simulations of the geometry from Fig. 2(b). These simulations are in excellent agreement with the experimental data of the density both for the nozzle and the capillary region. The shape of the density peak and the peak's height are confirmed as well as the estimated magnitude of density inside the capillary.

The creation of an exact model of this region is not possible due to the, for manufacturing reasons, bumpy surface of this area. Furthermore, the depth of the channel from the right inlet to the right vacuum is more than the $230 \mu \mathrm{m}$ inside the capillary. To take care of those facts the intersection inlet/vacuum on the right side was slightly increased in the 2D simulations compared to the real capillary.

An additional cross-check of the simulation with an independent CFD code FLUENT 6.3 was performed. The FLUENT simulation brought similar results as the OPENFOAM simulations, see Fig. 4(b). The density based k-omega-SST model was used as a solver for turbulences in this simulation. Slight differences of $6 \%$ in density peak height and capillary density can be seen. Besides this aberration, all parameters like peak shape and the overall density are approved by FLUENT simulation.

\section{CONCLUSION}

We present a new method towards a pressure measurement of a density profile in a narrow sapphire capillary for LWFA. The density dependence of Raman scattering was utilized to characterize the longitudinal density profile of a neutral gas in the laser-machined capillary. We succeeded in spectrally distinguishing density photons from stray photons and could detect a well pronounced density peak produced by the gas jet. Simulations were performed with two independent fluid dynamic codes; these simulations are in excellent agreement with our experimental observations. The experimental data show a density dip located to the right side of the gas jet which was not found in simulations. We are optimistic to further optimize this method in terms of density resolution and exposure time. The goal is to operate this method in a running LWFA experiment.

\section{ACKNOWLEDGMENTS}

We especially want to thank R. Kienberger for borrowing the VERDI laser and Zs. Major, I. Ahmad, and S. Trushin for helping with capillary machining. This work has been financially supported by the DFG through Transregio TR18 and supported by the DFG Cluster-ofExcellence Munich-Centre for Advanced Photonics MAP.

[1] W. P. Leemans et al., Nature Phys. 2, 696 (2006).

[2] S. Karsch et al., New J. Phys. 9, 415 (2007).

[3] J. Osterhoff et al., Phys. Rev. Lett. 101, 085002 (2008).

[4] S. Bulanov et al., Phys. Rev. E 58, R5257 (1998).

[5] G. Fubiani et al., Phys. Rev. E 73, 026402 (2006).

[6] A. J. Gonsalves et al., in Proceedings of the 23rd Particle Accelerator Conference, Vancouver, Canada, 2009 (IEEE, Piscataway, NJ, 2009).

[7] C. G. R. Geddes et al., Phys. Rev. Lett. 100, 215004 (2008).

[8] A. J. Gonsalves et al., Phys. Rev. Lett. 98, 025002 (2007).

[9] D. J. Spence et al., Opt. Lett. 24, 993 (1999).

[10] W. Demtröder, Experimental Physik 2 (Springer-Verlag, Berlin, 1999).

[11] E. D. Hinkley, Laser Monitoring of the Atmosphere (Springer, New York, 1976).

[12] http://www.openfoam.org. 